\title{
Correlation Studies in the Induced Mutant Population of Cluster Bean [Cyamopsis tetragonoloba (1.) Taub.]
}

\author{
N.M. Meghana ${ }^{1}$, R.K. Ramachandra ${ }^{2}$, Vishnuvardhana $^{1}$, B. Fakrudin ${ }^{1}$, \\ H.M. Pallavi ${ }^{2}$, M. Anjanappa ${ }^{1}$ and A. Harish ${ }^{3}$ \\ ${ }^{1}$ College of Horticulture, Bengaluru, Karnataka, India \\ ${ }^{2}$ College of Horticulture, Mysore, Karnataka, India \\ ${ }^{3}$ College of Horticulture, VCSG UUHF, Bharsar, Uttarakhand, India \\ *Corresponding author
}

\section{Keywords \\ Correlation studies, Mutant population, Cluster bean, Analysis of variance}

\section{Article Info}

Accepted:

04 February 2019

Available Online:

10 March 2019

\section{A B S T R A C T}

Variability studies provide information on the extent of improvement possible in different characters, but they do not throw light on the extent and nature of relationship existing between yield and various contributory characters. Character association or correlation is a measure of the degree of association between two characters. A knowledge regarding the association of various characters among themselves and with economic characters is necessary for making indirect selection for improvement of economic characters. The gamma radiation induced $190 \mathrm{M}_{4}$ mutant lines of cluster bean [Cyamopsis tetragonoloba (L.) Taub.] obtained from Centre for Biotechnological Research (CBR), College of Horticulture, Bengaluru were used for the field experimentation at College of Horticulture, Mysore. Such collected 190 M 4 mutants of cluster bean were investigated for various yield and its associated characters. The data was recorded on number of days to 50 per cent flowering, number of days to 50 per cent maturity, number of days to harvest, plant height, number of branches per plant, pod breadth, pod length, number of pods per cluster, number of clusters per plant, number of pods per plant, ten pods weight $(\mathrm{g})$, pod yield per plant $(\mathrm{g})$, seeds per pod, seed yield per plant (g), 100-seed weight (g). Analysis of variance revealed highly significant difference among the mutants for all the characters studied. Correlation revealed highly significant and positive association of vegetable pod yield and other yield component characters. A positive correlation for pod yield with days to 50 per cent maturity, pod length $(\mathrm{cm})$, pod width $(\mathrm{cm})$, days for harvest, pods per cluster, pod clusters per plant, pods per plant, 10 pods weight (g), pod yield per plant (g), seeds per pod, seed yield per plant $(\mathrm{g})$ and 100 seed weight $(\mathrm{g})$. A non-significant but positive correlation was also observed with plant height and number of branches per plant.

\section{Introduction}

Correlation provides information on the nature and extent of relationship among the characters. The estimates of correlation coefficient among the different characters indicate the extent of direction of association. Correlation between characters are important 
for three reasons in connection with (1) the genetic causes of correlation through the phenotypic action of genes, (2) the changes brought about by selection and (3) natural selection where the relation between quantitative traits and fitness is the primary agent that determines the genetic properties of that character in a natural population (Falconer, 1981). Yield is a complex character influenced by a large number of other component traits. A knowledge of the association between yield and its component traits and also between the component traits helps in improving the efficiency of selection.

Since there may not be gene for yield per se but for various yield components. Further, many of these yield contributing characters are interacted in desirable and undesirable direction. Hence, a knowledge regarding the association of various characters among themselves and with economic characters is necessary for making indirect selection for improvement of economic characters. Character association or correlation is a measure of the degree of association between two characters. Variability studies provide information on the extent of improvement possible in different characters, but they do not throw light on the extent and nature of relationship existing between yield and various contributory characters. As a rational approach for the improvement of yield, selection has to be made for components of yield.

\section{Materials and Methods}

The present experiment was carried out at the PG research block, College of Horticulture, Mysuru, during the year 2017- 18 involving the $190 \mathrm{M}_{4}$ mutant lines and three checks which were field evaluated in 8 blocks in an Augmented Block Design with repeated checks in each block. These mutants obtained from Centre for Biotechnology Research
(CBR) Department of BCI, COH, Bengaluru were planted at a spacing of $45 \times 25 \mathrm{~cm}$ on $14^{\text {th }}$ of June 2017 . The experiment was laid out following the recommended package of practices of UHS, Bagalkot for cluster bean (Anonymous 2016). The data was recorded on number of days to 50 per cent flowering, number of days to 50 per cent maturity, number of days to harvest, plant height, number of branches per plant, pod breadth, pod length, number of pods per cluster, number of clusters per plant, number of pods per plant, ten pods weight $(\mathrm{g})$, pod yield per plant (g), seeds per pod, seed yield per plant (g), 100-seed weight $(\mathrm{g})$. All the data collected were subjected to analysis for drawing the conclusion.

\section{Results and Discussion}

Extent and nature of relationship existing between yield and its various contributing character is important. As a rational approach for the improvement of yield, selection has to be made for components of yield, since there may not be gene for yield per se but for various yield components. Further, yield contributing traits are interacted in desirable and undesirable direction. Hence, a knowledge regarding the association of various characters among themselves and with economic characters is necessary for making indirect selection for improvement of economic characters. Character association or correlation is a measure of the degree of association between two characters in a population of individual. The estimates of correlation coefficient among the different characters indicate the extent of direction of association. Correlations between characters are important for three reasons. (1) The genetic causes of correlation through the phenotypic action of genes, (2) changes brought about by selection and (3) natural selection where the relation between quantitative traits and fitness is the primary 
agent that determines the genetic properties of that character in a natural population (Falconer, 1981). Yield is a complex character influenced by a large number of other component traits. A knowledge of the association between yield and its component traits and also between the component traits helps in improving the efficiency of selection.

In the present study the vegetable pod yield per plant exhibited positive and significant correlation with days to 50 per cent flowering, pod length, days to 50 per cent maturity, pod width, days for harvest, pods per cluster, pod cluster per plant, pods per plant, 10 pods weight, pod yield per plant, seeds per pod, seed yield per plant and 100 seed weight but it is positive and non-significant with plant height and number of branches per plant. These results were in confirmation with Mital and Thomas (1969), Brindha et al., (1996).

The seed yield per plant exhibited positive and significant correlation with days to 50 per cent flowering, pod length, days to 50 per cent maturity, pod width, days for harvest, pods per cluster, pod cluster per plant, pods per plant, 10 pods weight, pod yield per plant, seeds per pod, seed yield per plant and 100 seed weight but it is positive and non-significant with plant height and number of branches per plant. These results were in confirmation with Tyagi et al., (2000), Arumugarangarajan et al., (2000), Motior et al., (1997) and Saini et al., (2010). Plant height showed positive and significant correlation with number of branches per plant. It exhibited positive and non-significant correlation with days to 50 per cent flowering, pods per cluster, pod cluster per plant, pods per plant, 10 pods weight, pod yield per plant, seeds per pod, seed yield per plant and 100 seed weight. But negative and non-significant correlation was shown by pod length, days to 50 per cent maturity, pod width, days for harvest which was in agreement with the report of Singh et al.,
(2004). Number of branches per plant showed positive and non-significant correlation with pods per cluster, pods per plant, pod yield per plant, seed yield per plant. It exhibited negative and non-significant correlation with pod length, days to 50 per cent maturity, pod width, days for harvest, pod cluster per plant, 10 pods weight, seeds per pod, and 100 seed weight. This was supported by Sakrajitjana and Das (1983), Patel and Choudhary (2001).

Differences between GCV and PCV were also found to be less for all the eleven traits indicating that these traits were less affected by environmental fluctuations. High values of GCV over PCV suggested that there is a possibility of improvement through direct selection for these traits among landraces. Based on the above results, it is suggested that in different accessions, characters with high genotypic variability viz., plant height, number of branches per plant, number of clusters per plant, number of pods per cluster, seed size and hundred seed weight would be responsive to selection in the positive direction. Presence of variability in the population is a prerequisite for selection to be effective. Heritability estimates were high for all the characters studied. Similar results were obtained in previous study for the traits like days to 50 per cent flowering, number of primary branches per plant and hundred seed weight (91 per cent). If heritability of a character is very high, say 70 per cent or more, selection for such traits could be fairly easy. This is because there would be a close correspondence between the genotype and the phenotype due to the relatively small contribution of the environment to the phenotype according to Singh (2001). There was also a relatively high genetic advance as per cent mean for plant height, number of branches per plant, number of clusters per plant, number of pods per cluster, number of seeds per pod, pod length, seed size and hundred seed weight (Table 1 and 2). 
Table.1 Correlation coefficients for selected quantitative traits in M4 mutants of cluster bean

\begin{tabular}{|c|c|c|c|c|c|c|c|c|c|c|c|c|c|c|c|}
\hline & $\mathbf{X}_{1}$ & $\mathbf{X}_{2}$ & $\mathbf{X}_{\mathbf{3}}$ & $\mathbf{X}_{4}$ & $\mathbf{X}_{5}$ & $X_{6}$ & $\mathbf{X}_{7}$ & $\mathbf{X}_{8}$ & $\mathbf{X}_{9}$ & $\mathbf{X}_{10}$ & $\mathbf{X}_{11}$ & $X_{12}$ & $\mathbf{X}_{13}$ & $\mathbf{X}_{14}$ & $X_{15}$ \\
\hline $\mathbf{X}_{1}$ & 1.00 & $0.48 * *$ & $0.49 * *$ & $0.48 * *$ & $0.51 * *$ & $0.48 * *$ & $0.49 * *$ & $0.34 * *$ & $0.34 * *$ & $0.26 * *$ & $0.37 * *$ & $0.28 * *$ & $0.38 * *$ & $0.19 * *$ & $0.40 * *$ \\
\hline $\mathbf{X}_{2}$ & & 1.00 & $0.79 * *$ & -0.04 & -0.02 & -0.03 & -0.02 & 0.07 & 0.04 & 0.11 & 0.02 & 0.10 & 0.02 & 0.12 & 0.00 \\
\hline $\mathbf{X}_{3}$ & & & 1.00 & -0.06 & -0.06 & -0.04 & -0.05 & 0.00 & -0.01 & 0.04 & -0.06 & 0.02 & -0.05 & 0.03 & -0.08 \\
\hline$X_{4}$ & & & & 1.00 & $0.95 * *$ & $0.96 * *$ & $0.95 * *$ & $0.74 * *$ & $0.73 * *$ & $0.61 * *$ & $0.85 * *$ & $0.62 * *$ & $0.85^{* *}$ & $0.58^{* *}$ & $0.80 * *$ \\
\hline$X_{5}$ & & & & & 1.00 & $0.97 * *$ & $0.99 * *$ & $0.77 * *$ & $0.75^{* *}$ & $0.64 * *$ & $0.83 * *$ & $0.61 * *$ & $0.88 * *$ & $0.61 * *$ & $0.84 * *$ \\
\hline $\mathbf{X}_{6}$ & & & & & & 1.00 & $0.97 * *$ & $0.77 * *$ & $0.76 * *$ & $0.65^{* *}$ & $0.84 * *$ & $0.64 * *$ & $0.86^{* *}$ & $0.62 * *$ & $0.81 * *$ \\
\hline $\mathbf{X}_{7}$ & & & & & & & 1.00 & $0.78 * *$ & $0.76 * *$ & $0.64 * *$ & $0.83 * *$ & $0.61 * *$ & $0.88 * *$ & $0.62 * *$ & $0.84 * *$ \\
\hline $\mathbf{X}_{8}$ & & & & & & & & 1.00 & $0.63 * *$ & $0.83 * *$ & $0.67 * *$ & $0.75^{* *}$ & $0.68 * *$ & $0.74 * *$ & $0.72 * *$ \\
\hline $\mathbf{X}_{9}$ & & & & & & & & & 1.00 & $0.86^{* *}$ & $0.71 * *$ & $0.80 * *$ & $0.72 * *$ & $0.74 * *$ & $0.66^{* *}$ \\
\hline $\mathbf{X}_{10}$ & & & & & & & & & & 1.00 & $0.61 * *$ & $0.88 * *$ & $0.60 * *$ & $0.82 * *$ & $0.60 * *$ \\
\hline $\mathbf{X}_{11}$ & & & & & & & & & & & 1.00 & $0.68 * *$ & $0.84 * *$ & $0.65^{* *}$ & $0.73 * *$ \\
\hline $\mathbf{X}_{12}$ & & & & & & & & & & & & 1.00 & $0.59 * *$ & $0.74 * *$ & $0.58 * *$ \\
\hline $\mathbf{X}_{13}$ & & & & & & & & & & & & & 1.00 & $0.68^{* *}$ & $0.75^{* *}$ \\
\hline $\mathbf{X}_{14}$ & & & & & & & & & & & & & & 1.00 & $0.72 * *$ \\
\hline$X_{15}$ & & & & & & & & & & & & & & & 1.00 \\
\hline
\end{tabular}

* Correlation is significant at 5\% level of significance

$\mathrm{X}_{1}=$ Days for $50 \%$ flowering

$\mathrm{X}_{2}=$ Plant height $(\mathrm{cm})$

$\mathrm{X}_{3}=$ Branches/plant

$\mathrm{X}_{4}=$ Pod length $(\mathrm{cm})$

$\mathrm{X}_{5}=$ Days for $50 \%$ maturity

** Correlation is significant at $1 \%$ level of significance

$\mathrm{X}_{6}=$ Pod width $(\mathrm{cm})$

$\mathrm{X}_{7}=$ Days for harvest

$\mathrm{X}_{8}=$ Pods/cluster

$\mathrm{X}_{9}=$ Pod clusters/plant

$\mathrm{X}_{10}=$ Pods/plant
$\mathrm{X}_{11}=$ Ten pods weight $(\mathrm{g})$

$\mathrm{X}_{12}=$ Pod yield/plant $(\mathrm{g})$

$\mathrm{X}_{13}=$ Seeds/pod

$\mathrm{X}_{14}=$ Seed yield/plant $(\mathrm{g})$

$X_{15}=100$ seed weight $(\mathrm{g})$ 
Table.2 Estimates of variability for various quantitative traits among the M4 mutants of cluster bean

\begin{tabular}{|c|c|c|c|c|c|c|c|c|}
\hline & \multirow[t]{2}{*}{ Traits } & \multirow[t]{2}{*}{ Mean } & \multicolumn{2}{|l|}{ Range } & \multirow{2}{*}{$\begin{array}{l}\text { PCV } \\
(\%)\end{array}$} & \multirow{2}{*}{$\begin{array}{l}\text { GCV } \\
(\%)\end{array}$} & \multirow[t]{2}{*}{$h^{2}$} & \multirow{2}{*}{$\begin{array}{l}\text { GA as } \\
\% \text { of } \\
\text { mean }\end{array}$} \\
\hline & & & Minimum & Maximum & & & & \\
\hline & & & 26.00 & 38.00 & & & & \\
\hline 1 & Plant height $(\mathrm{cm})$ & 54.85 & 33.80 & 96.60 & 29.57 & 25.13 & 72.20 & 43.63 \\
\hline 2 & Branches/plant & 12.27 & 6.80 & 23.00 & 31.68 & 25.08 & 62.70 & 41.23 \\
\hline 3 & Pod length $(\mathrm{cm})$ & 9.96 & 0.00 & 13.85 & 33.76 & 32.36 & 91.80 & 63.74 \\
\hline 4 & $\begin{array}{l}\text { Days for } 50 \% \\
\text { maturity }\end{array}$ & 48.00 & 0.00 & 58.00 & 31.75 & 31.61 & 99.10 & 64.80 \\
\hline 5 & Pod width $(\mathrm{cm})$ & 0.92 & 0.00 & 1.17 & 33.64 & 31.15 & 91.10 & 62.15 \\
\hline 6 & Days for harvest & 61.58 & 0.00 & 75.00 & 31.66 & 31.54 & 99.30 & 64.70 \\
\hline 7 & Pods/cluster & 6.07 & 0.00 & 11.50 & 39.59 & 39.52 & 99.60 & 81.15 \\
\hline 8 & $\begin{array}{l}\text { Pod } \\
\text { clusters/plant }\end{array}$ & 11.91 & 0.00 & 20.10 & 40.49 & 40.41 & 99.60 & 83.10 \\
\hline 9 & Pods/plant & 79.46 & 0.00 & 164.05 & 47.18 & 47.06 & 99.50 & 96.76 \\
\hline 10 & $\begin{array}{l}\text { Ten pods weight } \\
(\mathrm{g})\end{array}$ & 36.76 & 0.00 & 62.70 & 37.72 & 35.87 & 90.40 & 70.35 \\
\hline 11 & $\begin{array}{l}\text { Pod yield/plant } \\
\text { (g) }\end{array}$ & 206.62 & 0.00 & 368.72 & 49.17 & 49.05 & 99.50 & 99.10 \\
\hline 12 & Seeds/pod & 5.77 & 0.00 & 8.40 & 36.72 & 34.40 & 87.80 & 65.33 \\
\hline 13 & $\begin{array}{l}\text { Seed yield/plant } \\
(\mathrm{g})\end{array}$ & 13.33 & 0.00 & 29.16 & 48.68 & 48.50 & 99.30 & 98.94 \\
\hline 14 & $\begin{array}{l}100 \text { seed weight } \\
(\mathrm{g})\end{array}$ & 4.33 & 0.00 & 7.92 & 37.45 & 37.27 & 99.00 & 76.72 \\
\hline
\end{tabular}


Appendix: The details of various M4 cluster bean mutants used in the investigation

\begin{tabular}{|c|c|c|c|c|c|c|c|c|}
\hline Sl. No. & Reference code & Mutant name & Sl. No. & $\begin{array}{l}\text { Reference } \\
\text { code }\end{array}$ & Mutant name & Sl. No. & Reference code & Mutant name \\
\hline & $\mathrm{A}_{1}$ & $80-17-1$ & 2. & $\mathrm{~B}_{63}$ & 100-ST80-17 & 3. & $\mathrm{D}_{125}$ & $100-16-6$ \\
\hline 4. & $\mathrm{~A}_{2}$ & $80-3-2$ & 5. & $\mathrm{~B}_{64}$ & $100-10-17$ & 6. & $\mathrm{D}_{126}$ & $100-5-12$ \\
\hline 7. & $\mathrm{~A}_{3}$ & $80-24-3$ & 8. & $\mathrm{~B}_{65}$ & $100-5-7$ & 9. & $\mathrm{D}_{127}$ & $100-5-15$ \\
\hline 10. & $\mathrm{~A}_{4}$ & $80-20-3$ & 11. & $\mathrm{~B}_{66}$ & $100-10-4$ & 12. & $\mathrm{D}_{128}$ & $100-5-8$ \\
\hline 13. & $\mathrm{~A}_{5} \mathrm{~S}$ & $80-28-3$ & 14. & $\mathrm{~B}_{67}$ & $100-5-14$ & 15. & $\mathrm{D}_{129}$ & $100-5-10$ \\
\hline 16. & $\mathrm{~A}_{6}$ & $80-6-6$ & 17. & $\mathrm{~B}_{68}$ & $100-1-2$ & 18. & $\mathrm{D}_{130}$ & $100-22-7$ \\
\hline 19. & $\mathrm{~A}_{7}$ & $80-9-5$ & 20. & $\mathrm{~B}_{69}$ & 100-ST80-23 & 21. & $\mathrm{D}_{131}$ & $100-25-2$ \\
\hline 22. & $\mathrm{~A}_{8}$ & $80-6-5$ & 23. & $\mathrm{~B}_{70}$ & $100-1-10$ & 24. & $\mathrm{D}_{132}$ & $100-10-3$ \\
\hline 25. & $\mathrm{~A}_{9}$ & $80-3-4$ & 26. & $\mathrm{~B}_{71}$ & $100-1-5$ & 27. & $\mathrm{D}_{133}$ & $100-25-3$ \\
\hline 28. & $\mathrm{~A}_{10}$ & $80-17-5$ & 29. & $\mathrm{~B}_{72}$ & $100-22-1$ & 30. & $\mathrm{D}_{134}$ & $100-2-3$ \\
\hline 31. & $\mathrm{~A}_{11}$ & $80-19-2$ & 32. & $\mathrm{~B}_{73}$ & $100-21-2$ & 33. & $\mathrm{D}_{135}$ & $100-16-5$ \\
\hline 34. & $\mathrm{~A}_{12}$ & $80-17-2$ & 35. & $\mathrm{~B}_{74}$ & $100-2-4$ & 36. & $\mathrm{D}_{136} \mathrm{~S}$ & $100-26-6$ \\
\hline 37. & $\mathrm{~A}_{13}$ & $80-18-17$ & 38. & $\mathrm{~B}_{75}$ & 100-ST80-27 & 39. & $\mathrm{D}_{137}$ & $100-26-1$ \\
\hline 40. & $\mathrm{~A}_{14}$ & $80-27-1$ & 41. & $\mathrm{~B}_{76}$ & 100-ST80-3 & 42. & $\mathrm{D}_{138}$ & $100-5-9$ \\
\hline 43. & $\mathrm{~A}_{15}$ & $80-17-3$ & 44. & $\mathrm{~B}_{77}$ & $100-22-3$ & 45. & $\mathrm{D}_{139}$ & $100-25-5$ \\
\hline 46. & $\mathrm{~A}_{16} \mathrm{~S}$ & $80-18-3$ & 47. & $\mathrm{~B}_{78}$ & $100-5-1$ & 48. & $\mathrm{D}_{140}$ & $100-5-2$ \\
\hline 49. & $\mathrm{~A}_{17}$ & $80-9-2$ & 50. & $\mathrm{~B}_{79}$ & $100-75-7$ & 51. & $\mathrm{D}_{141} \mathbf{S}$ & $100-26-4$ \\
\hline 52. & $\mathrm{~A}_{18}$ & $80-18-7$ & 53. & $\mathrm{C}_{80}$ & $100-5-13$ & 54. & $\mathrm{D}_{142}$ & $100-10-6$ \\
\hline 55. & $\mathrm{~A}_{19}$ & $80-3-1$ & 56. & $\mathrm{C}_{81}$ & $100-2-5$ & 57. & $\mathrm{D}_{143}$ & $100-21-1$ \\
\hline 58. & $\mathrm{~A}_{20}$ & $80-18-2$ & 59. & $\mathrm{C}_{82}$ & $100-2-7$ & 60. & $\mathrm{D}_{144}$ & $100-22-8$ \\
\hline 61. & $\mathrm{~A}_{21}$ & $80-3-3$ & 62. & $\mathrm{C}_{83}$ & $100-21-5$ & 63. & $\mathrm{D}_{145}$ & $100-10-1$ \\
\hline 64. & $\mathrm{~A}_{22}$ & $80-6-2$ & 65. & $\mathrm{C}_{84}$ & $100-22-6$ & 66. & $\mathrm{D}_{146}$ & $100-1-8$ \\
\hline 67. & $\mathrm{~A}_{23} \mathrm{~S}$ & $80-9-4$ & 68. & $\mathrm{C}_{85}$ & $100-2-6$ & 69. & $\mathrm{D}_{147}$ & $100-25-9$ \\
\hline 70. & $\mathrm{~A}_{24}$ & $80-28-7$ & 71. & $\mathrm{C}_{86}$ & $100-10-8$ & 72. & $\mathrm{D}_{148}$ & $100-2-1$ \\
\hline 73. & $\mathrm{~A}_{25}$ & $80-28-6$ & 74. & $\mathrm{C}_{87}$ & $100-26-5$ & 75. & $\mathrm{D}_{149}$ & $100-1-9$ \\
\hline 76. & $\mathrm{~A}_{26}$ & $80-23-3$ & 77. & $\mathrm{C}_{88}$ & $100-1-4$ & 78. & $\mathrm{D}_{150}$ & $100-5-3$ \\
\hline 79. & $\mathrm{~A}_{27}$ & 80-19-1 & 80. & $\mathrm{C}_{89}$ & $100-16-7$ & 81. & $\mathrm{D}_{151}$ & $100-5-16$ \\
\hline 82. & $\mathrm{~A}_{28}$ & $80-20-6$ & 83. & $\mathrm{C}_{90}$ & $100-26-8$ & 84. & $\mathrm{D}_{152}$ & $100-25-1$ \\
\hline 85. & $\mathrm{~A}_{29}$ & $80-28-1$ & 86. & $\mathrm{C}_{91}$ & $100-16-3$ & 87. & $\mathrm{D}_{153}$ & $100-5-17$ \\
\hline 88. & $\mathrm{~A}_{30}$ & $80-24-5$ & 89. & $\mathrm{C}_{92}$ & $80-24-1$ & 90. & $\mathrm{D}_{154}$ & $100-10-2$ \\
\hline 91. & $\mathrm{~A}_{31}$ & $80-28-2$ & 92. & $\mathrm{C}_{93} \mathrm{~S}$ & $80-9-3$ & 93. & $\mathrm{D}_{155}$ & $100-2-2$ \\
\hline
\end{tabular}




\begin{tabular}{|c|c|c|c|c|c|c|c|c|}
\hline 94. & $\mathrm{~A}_{32}$ & $80-20-4$ & 95. & $\mathrm{C}_{94} \mathrm{~S}$ & $80-6-4$ & 96. & $\mathrm{D}_{156}$ & $100-\mathrm{ST} 80-22$ \\
\hline 97. & $\mathrm{~A}_{33} \mathbf{N P}$ & $80-27-5$ & 98. & $\mathrm{C}_{95} \mathrm{NP}$ & $80-18-5$ & 99. & $\mathrm{D}_{157}$ & 100-ST80-1 \\
\hline 100. & $\mathrm{~A}_{34}$ & $80-20-2$ & 101. & $\mathrm{C}_{96}$ & $80-6-3$ & 102. & $\mathrm{D}_{158}$ & 100-ST80-4 \\
\hline 103. & $\mathrm{~A}_{35}$ & $80-20-1$ & 104. & $\mathrm{C}_{97}$ & $80-17-4$ & 105. & $\mathrm{D}_{159} \mathbf{N P}$ & $100-1-7$ \\
\hline 106. & $\mathrm{~A}_{36}$ & $80-27-2$ & 107. & $\mathrm{C}_{98}$ & $80-9-1$ & 108. & $\mathrm{D}_{160}$ & $100-25-9$ \\
\hline 109. & $\mathrm{~A}_{37}$ & $80-23-2$ & 110. & $\mathrm{C}_{99}$ & $80-3-5$ & 111. & $\mathrm{D}_{161}$ & $100-10-5$ \\
\hline 112. & $\mathrm{~A}_{38}$ & $80-27-3$ & 113. & $\mathrm{C}_{100}$ & 80-P58-7 & 114. & $\mathrm{D}_{162}$ & $100-25-4$ \\
\hline 115. & $\mathrm{~A}_{39}$ & $80-27-4$ & 116. & $\mathrm{C}_{101}$ & 80-P58-9 & 117. & $\mathrm{D}_{163}$ & $100-1-1$ \\
\hline 118. & $\mathrm{~B}_{40}$ & $100-21-3$ & 119. & $\mathrm{C}_{102} \mathrm{~S}$ & 80-P58-8 & 120. & $\mathrm{D}_{164}$ & $100-16-1$ \\
\hline 121. & $\mathrm{~B}_{41}$ & 100-ST80-14 & 122. & $\mathrm{C}_{103}$ & 100-P3-80-1 & 123. & $\mathrm{D}_{165}$ & $100-16-2$ \\
\hline 124. & $\mathrm{~B}_{42}$ & $100-21-4$ & 125. & $\mathrm{C}_{104}$ & $100-P 3-80-4$ & 126. & $\mathrm{E}_{166}$ & $100-M S 2-5$ \\
\hline 127. & $\mathrm{~B}_{43}$ & 100-ST80-8 & 128. & $\mathrm{C}_{105}$ & 100-P3-80-2 & 129. & $\mathrm{E}_{167}$ & 100-MS2-3 \\
\hline 130. & $\mathrm{~B}_{44}$ & 100-ST80-28 & 131. & $\mathrm{C}_{106}$ & 100-P3-80-3 & 132. & $\mathrm{E}_{168}$ & 100-MS2-1 \\
\hline 133. & $\mathrm{~B}_{45}$ & 100-ST80-12 & 134. & $\mathrm{C}_{107}$ & $100-A 80-4$ & 135. & $\mathrm{E}_{169}$ & 100-MS2-2 \\
\hline 136. & $\mathrm{~B}_{46}$ & 100-ST80-20 & 137. & $\mathrm{C}_{108}$ & 100-A80-2 & 138. & $\mathrm{E}_{170}$ & 100-MS2-4 \\
\hline 139. & $\mathrm{~B}_{47}$ & 100-ST80-16 & 140. & $\mathrm{C}_{109} \mathrm{NP}$ & 100-A80-1 & 141. & $\mathrm{E}_{171}$ & 100-MS2-6 \\
\hline 142. & $\mathrm{~B}_{48}$ & 100-ST80-24 & 143. & $\mathrm{C}_{110}$ & $100-A 80-5$ & 144. & $\mathrm{E}_{172}$ & 80-P58-11 \\
\hline 145. & $\mathrm{~B}_{49}$ & 100-ST80-7 & 146. & $\mathrm{C}_{111}$ & 100-A $80-3$ & 147. & $\mathrm{E}_{173}$ & 80-P58-3 \\
\hline 148. & $\mathrm{~B}_{50}$ & 100-ST80-13 & 149. & $\mathrm{C}_{112}$ & 100-PNB-1 & 150. & $\mathrm{E}_{174}$ & 80-P58-4 \\
\hline 151. & $\mathrm{~B}_{51}$ & $100-25-8$ & 152. & $\mathrm{C}_{113}$ & 100-PNB-2 & 153. & $\mathrm{E}_{175}$ & 80-P58-10 \\
\hline 154. & $\mathrm{~B}_{52} \mathrm{~S}$ & $100-5-19$ & 155. & $\mathrm{C}_{114}$ & 100-PNB-3 & 156. & $\mathrm{E}_{176}$ & 80-P58-1 \\
\hline 157. & $\mathrm{~B}_{53}$ & 100-ST80-21 & 158. & $\mathrm{C}_{115}$ & 100-PNB-4 & 159. & $\mathrm{E}_{177}$ & 80-P58-5 \\
\hline 160. & $\mathrm{~B}_{54}$ & 100-ST80-15 & 161. & $\mathrm{C}_{116}$ & P58-7 & 162. & $\mathrm{E}_{178}$ & 80-P58-2 \\
\hline 163. & $\mathrm{~B}_{55}$ & 100-ST80-10 & 164. & $\mathrm{C}_{117}$ & 100-ST80-5 & 165. & $\mathrm{E}_{179}$ & 80-P58-6 \\
\hline 166. & $\mathrm{~B}_{56}$ & $100-22-2$ & 167. & $\mathrm{C}_{118}$ & 100-ST80-9 & 168. & $\mathrm{E}_{180}$ & 80-MN2-2 \\
\hline 169. & $\mathrm{~B}_{57}$ & 100-ST80-19 & 170. & $\mathrm{C}_{119}$ & 100-ST80-3 & 171. & $\mathrm{E}_{181}$ & 80-MN2-6 \\
\hline 172. & $\mathrm{~B}_{58}$ & $100-10-11$ & 173. & $\mathrm{C}_{120} \mathrm{NP}$ & 100-ST80-2 & 174. & $\mathrm{E}_{182}$ & 80-MN2-7 \\
\hline 175. & $\mathrm{~B}_{59}$ & 100-ST80-25 & 176. & $\mathrm{C}_{121}$ & 80-MN3-6 & 177. & $\mathrm{E}_{183}$ & 80-MN2-1 \\
\hline 178. & $\mathrm{~B}_{60} \mathrm{~S}$ & 100-ST80-18 & 179. & $\mathrm{C}_{122}$ & 80-MN3-3 & 180. & $\mathrm{E}_{184}$ & 80-MN2-4 \\
\hline 181. & $\mathrm{~B}_{61} \mathrm{~S}$ & 100-ST80-6 & 182. & $\mathrm{D}_{123}$ & $100-1-6$ & 183. & $\mathrm{E}_{185}$ & 80-MN2-5 \\
\hline 184. & $\mathrm{~B}_{62}$ & 100-ST80-11 & 185. & $\mathrm{D}_{124} \mathbf{S}$ & $100-26-7$ & 186. & $\mathrm{E}_{186}$ & 80-MN3-5 \\
\hline 187. & $\mathrm{E}_{188}$ & 80-MN3-2 & 188. & $\mathrm{E}_{189}$ & 80-MN3-1 & 189. & $\mathrm{E}_{187}$ & 80-MN3-7 \\
\hline 190. & $\mathrm{E}_{190}$ & 80-MN3-3 & 191. & & & 192. & & \\
\hline
\end{tabular}


It was also reported high GAM coupled with high heritability for plant height, seed yield, hundred seed weight, harvest index and daily production rate indicated heritable nature of variation and scope for selection for these traits among the vegetable soybean genotypes. Johnson et al., (1955) suggested that high heritability combined with high genetic advance as per cent mean is indicative of additive gene action and selection based on these parameters would be more reliable.

It is concluded as correlation revealed highly significant and positive association of vegetable pod yield and other yield component characters. A positive correlation for pod yield with days to 50 per cent maturity, pod length $(\mathrm{cm})$, pod width $(\mathrm{cm})$, days for harvest, pods per cluster, pod clusters per plant, pods per plant, 10 pods weight $(\mathrm{g})$, pod yield per plant $(\mathrm{g})$, seeds per pod, seed yield per plant (g) and 100 seed weight $(\mathrm{g})$. A non-significant but positive correlation was also observed with plant height and number of branches per plant.

\section{Acknowledgements}

Authors are thankful to Dean Dr. G. Janardhan and Farm superintendent Dr. Yathindra at College of Horticulture, Mysuru, Karnataka, Dr. Mukesh L. Chavan, Profesor and Head Department of Biotchnology and Crop Improvement, Dr. V. Nachegowada, Director of Research UHS Bagalkot, Dr. N. Basavaraja Dean ( Post Graduate Studies ) at UHS Bagalkot for their support during the time of this investigation, Authors are also thankful to all the III B.Sc.(Horti) students of College of Horticulture, Mysore for extending timely help, The first author dedicated all her success to her beloved parents. Her journey in this world paves through the path curved by her lovable father Sri. Manjappa, B. The first author also found immeasurable amount of sacrifices, love, affection, support and concern from her mother Smt. Kamala, O., The first authors gratitude is beyond words to her lovely sister Monika, N. M. and dearest brother Siddarth, N. M. (ricky) for their unselfish struggle towards her wellbeing and attainment of perfection in carrier goals.

\section{References}

Arumugarangarajan, P., Jebaraj, S. and Backiyarani, S., 2000, Relationship among major economic characters in cluster bean (Cyamopsis tetragonoloba (L.) Taub.). South Indian Hort., 48(1-6): 64-68.

Brindha, S., Ponnuswamy, V. and Thamburaj, S., 1996, Correlation studies in cluster bean [Cyamopsis tetragonoloba (L.) Taub.]. South Indian Hort., 45(1-2): 5960.

Falconer, D. S., 1981, Introduction to quantitative genetics (2 Ed.). Longman Inc., New York 1: 340.

Johnson, H. W., Robinson, H. F. and Comstock, R. S., 1955, Estimation of genetic and environmental variability in soybean. Agron J., 41: 314-318.

Mital, S. P. and Thomas, T. A., 1969, Correlations and selection indices in improvement of seed yield in guar. Indian J. Genet., 29(1): 11-17.

Motior, M. R., Wanmohamad, W. O., Wong, K. C. and Shamsuddin, Z. H., 1997, Dry matter accumulation and partitioning in field grown winged bean under various support systems. Indian $\mathrm{J}$. Pl. Physi., 2(3): 217-220.

Patel, B. V. and Chaudhary, F. P., 2001, Component analysis of yield in cluster bean. Forage Res., 27(2): 123-125Saini et al., (2010).

Sakrajitjana, S. M. G. and Das, N. D., 1983, Genetic variability and correlation studies in cowpea. Mysore J. Agric. Sci., 18: 96-106.

Singh, J. V., Chander, S. and Punia, A., 2002, 
Studies on characters association in cluster bean [Cyamopsis tetragonoloba (L.) Taub. ]. J. Plant Improv., 4(1): 7174.

Tyagi, D. C., Nirmalkumar and Agarwal, M.
C., 2000, Genetic variability and association of component characters for seed yield in cowpea [Vigna unguiculata (L.) Warp.]. Legume Res., 23(2): 97-101

\section{How to cite this article:}

Meghana, N.M., R.K. Ramachandra, Vishnuvardhana, B. Fakrudin, H.M. Pallavi, M. Anjanappa and Harish, A. 2019. Correlation Studies in the Induced Mutant Population of Cluster Bean [Cyamopsis tetragonoloba (1.) Taub.]. Int.J.Curr.Microbiol.App.Sci. 8(03): 121129. doi: https://doi.org/10.20546/ijcmas.2019.803.017 